\title{
The initial gas-phase sulfur abundance in the Orion Molecular Cloud from sulfur radio recombination lines ${ }^{\star}$
}

\author{
Javier R. Goicoechea and Sara Cuadrado
}

\author{
Instituto de Física Fundamental (CSIC), Calle Serrano 121-123, 28006 Madrid, Spain \\ e-mail: javier.r.goicoechea@csic.es
}

Received 8 February 2021 / Accepted 2 March 2021

\begin{abstract}
The abundances of chemical elements and their depletion factors are essential parameters for understanding the composition of the gas and dust that are ultimately incorporated into stars and planets. Sulfur is an abundant but peculiar element in the sense that, despite being less volatile than other elements (e.g., carbon), it is not a major constituent of dust grains in diffuse interstellar clouds. Here, we determine the gas-phase carbon-to-sulfur abundance ratio, $[\mathrm{C}] /[\mathrm{S}]$, and the $[\mathrm{S}]$ in a dense star-forming cloud from new radio recombination lines (RRLs) detected with the Yebes $40 \mathrm{~m}$ telescope - at relatively high frequencies $(\sim 40 \mathrm{GHz} \simeq 7 \mathrm{~mm})$ and angular resolutions (down to 36") - in the Orion Bar, a rim of the Orion Molecular Cloud (OMC). We detect nine Cn $\alpha$ RRLs (with $n=51-59$ ) as well as nine narrow line features separated from the Cn $\alpha$ lines by $\delta v=-8.4 \pm 0.3 \mathrm{~km} \mathrm{~s}^{-1}$. Based on this velocity separation, we assign these features to sulfur RRLs, with little contribution of RRLs from the more condensable elements $\mathrm{Mg}, \mathrm{Si}$, or Fe. Sulfur RRLs lines trace the photodissociation region of the OMC. In these neutral gas layers, up to $A_{V} \simeq 4$, the ions $\mathrm{C}^{+}$and $\mathrm{S}^{+}$lock in most of the $\mathrm{C}$ and $\mathrm{S}$ gas-phase reservoir. We determine a relative abundance of $[\mathrm{C}]_{\text {Ori }} /[\mathrm{S}]_{\text {Ori }}=10.4 \pm 0.6$ and, adopting the same $[\mathrm{C}]_{\text {Ori }}$ measured in the translucent gas toward $\operatorname{star} \theta^{1}$ Ori $\mathrm{B}$, an absolute abundance of $[\mathrm{S}]_{\text {Ori }}=(1.4 \pm 0.4) \cdot 10^{-5}$. This value is consistent with emission models of the observed sulfur RRLs if $N\left(\mathrm{~S}^{+}\right) \simeq 7 \cdot 10^{17} \mathrm{~cm}^{-2}$ (beam-averaged). The [S] Ori is the "initial" sulfur abundance in the OMC, before an undetermined fraction of the $[\mathrm{S}]_{\text {Ori }}$ goes into molecules and ice mantles in the cloud interior. The inferred abundance $[\mathrm{S}]_{\text {Ori }}$ matches the solar abundance, thus implying that there is little depletion of sulfur onto rocky dust grains, with $D(\mathrm{~S})=0.0 \pm 0.2 \mathrm{dex}$.
\end{abstract}

Key words. ISM: abundances - radio lines: ISM - line: identification - photon-dominated region

\section{Introduction}

Ultraviolet (UV) line absorption studies show that the gas-phase abundance of certain elements $(\mathrm{Si}, \mathrm{Mg}, \mathrm{Fe}, \mathrm{Ca}, \mathrm{Ti}$, etc.) are highly depleted in diffuse $\left(A_{V} \lesssim 1\right)$ and translucent $\left(A_{V} \gtrsim 1\right)$ lowdensity clouds of the interstellar medium (ISM) ${ }^{1}$, with $D(\mathrm{X})$ ranging from -1 to -3 dex (Savage \& Sembach 1996; Sofia 2004; Jenkins 2009). This is consistent with their incorporation into (mainly) silicate grains (Mathis 1990; Draine 2003). The inferred depletion factors of much more volatile elements, such as carbon, are lower but still significant, $D(\mathrm{C}) \gtrsim-0.5$ dex.

Sulfur is on the top ten list of the most abundant elements ${ }^{1}$, with $[\mathrm{S}]_{\odot}=(1.4 \pm 0.1) \cdot 10^{-5}$, but it is an unusual element in the sense that almost all of the sulfur in diffuse clouds remains in the gas phase (Fitzpatrick \& Spitzer 1994; Howk et al. 2006). How-

\footnotetext{
* Based on observations with the $40 \mathrm{~m}$ radio telescope of the Spanish National Geographic Institute (IGN) at Yebes Observatory (project 19A005). Yebes Observatory thanks the ERC for funding support under grant ERC-2013-Syg-610256-NANOCOSMOS.

1 We refer to the gas-phase abundance "[X]" with respect to $\mathrm{H}$ nuclei as the gas column density ratio $[\mathrm{X}]=N(\mathrm{X}) / N_{\mathrm{H}}$, where $N_{\mathrm{H}}=N(\mathrm{H})+2 N\left(\mathrm{H}_{2}\right)$ in molecular clouds. We denote the "solar" or "bulk" abundance of element X in the Sun (i.e., the current photospheric abundances corrected for diffusion) as $[\mathrm{X}]_{\odot}$, with $[\mathrm{C}]_{\odot}=2.9 \cdot 10^{-4}$, $[\mathrm{Fe}]_{\odot}=3.5 \cdot 10^{-5},[\mathrm{Mg}]_{\odot}=4.4 \cdot 10^{-5}$, and $[\mathrm{Si}]_{\odot}=3.6 \cdot 10^{-5}$ (Asplund et al. $2009)$. We took these $[X]_{\odot}$ as the cosmic abundances and used the logarithmic depletion factor defined as $D(\mathrm{X})=\log [\mathrm{X}]-\log [\mathrm{X}]_{\odot}$.
}

ever, the relevant UV S II lines saturate, and thus the determination of [S] in neutral atomic $\mathrm{HI}$ and translucent molecular clouds may be uncertain (Federman et al. 1993; Sofia 2004).

Sulfur plays an important role in stellar nucleosynthesis; it is mainly produced in massive stars (e.g., Perdigon et al. 2021), in star formation, and in astrochemistry (e.g., Fuente et al. 2017; Shingledecker et al. 2020). Inside star-forming clouds, an undetermined fraction of sulfur gradually converts into molecules and ice mantles (e.g., Goicoechea et al. 2021), and yet the initial [S] in these dense molecular clouds is poorly constrained.

Radio recombination lines (RRLs) provide a powerful tool for studying star-forming regions independently of dust obscuration. Hydrogen and He RRLs are extensively used to constrain the morphology and physical conditions $\left(T_{\mathrm{e}}\right.$ and $\left.n_{\mathrm{e}}\right)$ of fully ionized H II regions (e.g., Churchwell et al. 1978). However, only stellar far-UV (FUV) photons, with energies below $13.6 \mathrm{eV}$, permeate the rims of molecular clouds, so-called photodissociation regions (PDRs; Hollenbach \& Tielens 1999). In the first layers of a PDR, the dominant state of elements with ionization potential (IP) below $\mathrm{H}$ is singly ionized: $\mathrm{C}^{+}(11.3 \mathrm{eV})$, $\mathrm{S}^{+}(10.4 \mathrm{eV}), \mathrm{Si}^{+}(8.2 \mathrm{eV}), \mathrm{Fe}^{+}(7.9 \mathrm{eV})$, or $\mathrm{Mg}^{+}(7.6 \mathrm{eV})$. Indeed, the narrower carbon RRLs probe these neutral PDRs adjacent to H II regions (Ball et al. 1970; Natta et al. 1994; Wyrowski et al. 1997; Salas et al. 2019; Cuadrado et al. 2019). Carbon RRLs have historically been detected in the centimeter (cm) domain: $\sim 8.6 \mathrm{GHz}$ for the $\mathrm{C} 91 \alpha$ line (where $\alpha$ stands for $\Delta n=1$ transitions). Compared to the fine-structure ${ }^{2} P_{3 / 2}-{ }^{2} P_{1 / 2}$ 
line of $\mathrm{C}^{+}$, the very important far-infrared [C II] $158 \mu \mathrm{m}$ cooling line (Hollenbach \& Tielens 1999), carbon RRLs are optically thin and their intensity is proportional to $n_{\mathrm{e}}^{2} T_{\mathrm{e}}^{-1.5}$. That is, they have different excitation properties than the [C II] $158 \mu \mathrm{m}$ line (e.g., Natta et al. 1994; Salas et al. 2019).

The ${ }^{4} S$ ground-electronic state of sulfur ions does not have low-lying fine-structure splittings. Hence, $\mathrm{S}^{+}$in neutral gas cannot be detected ${ }^{2}$ by far-infrared observations. Instead, sulfur RRLs, although much less studied, can be detected, typically after long-time integrations, and probe the " $\mathrm{S}^{+}$layer" of a $\mathrm{PDR}^{3}$. Early observations with $\mathrm{cm}$-wave single-dish radio telescopes, at several-arcminute resolution, allowed the detection of emission features at the high-frequency shoulder of carbon RRLs. These features were associated with sulfur RRLs but can correspond to a superposition of RRLs from low-IP elements heavier than carbon (Chaisson et al. 1972; Chaisson \& Lada 1974; Pankonin \& Walmsley 1978; Falgarone et al. 1978; Silverglate 1984; Vallee 1989; Smirnov et al. 1995).

In this Letter we present the first detection of sulfur RRLs in the Orion Bar (e.g., Tielens et al. 1993; Wyrowski et al. 1997; Walmsley et al. 2000; Goicoechea et al. 2016). This prototypical dense PDR (see Fig. 1) is a nearly edge-on interface of the Huygens $\mathrm{H}$ II region that is photoionized by the Trapezium massive stars $\theta^{1}$ Ori (O'Dell 2001) and the Orion Molecular Cloud (OMC) - the closest region to host ongoing massive-star formation (e.g., Genzel \& Stutzki 1989; Pabst et al. 2020).

\section{Observations}

We used the Yebes $40 \mathrm{~m}$ radio telescope at Yebes Observatory (Guadalajara, Spain) to observe the Bar. The observed position encompasses the dissociation front (DF), hereafter the DF position, at $\alpha_{2000}=05^{\mathrm{h}} 35^{\mathrm{m}} 20.8^{\mathrm{s}}, \delta_{2000}=-05^{\circ} 25^{\prime} 17.0^{\prime \prime}$. We observed the complete $Q$ band, from $31.1 \mathrm{GHz}$ to $50.4 \mathrm{GHz}$, using the new high-electron-mobility transistor (HEMT) $Q$ band receiver and fast Fourier transform spectrometers. These cover $18 \mathrm{GHz}$ of instantaneous bandwidth per polarization at a spectral resolution of $38 \mathrm{kHz}\left(\sim 0.3 \mathrm{~km} \mathrm{~s}^{-1}\right.$ at $\left.\sim 40 \mathrm{GHz}\right)$. We employed two frequency setups of slightly different central frequencies to identify any spurious line. The main beam efficiency varies from 0.6 at $32 \mathrm{GHz}$ to 0.43 at $48 \mathrm{GHz}$, and the half power beam width (HPBW) at these frequencies ranges from $\theta_{\text {FHWM }} \sim 54^{\prime \prime}$ to $36^{\prime \prime}$ (Tercero et al. 2021). We used the position switching observing mode, with a reference position at an offset $\left(-600^{\prime \prime}, 0^{\prime \prime}\right)$. The total integration time was $\sim 8 \mathrm{~h}$ under winter conditions $(\sim 8 \mathrm{~mm}$ of precipitable water vapor). We reduced and analyzed the data using the CLASS software of GILDAS. The achieved root mean square (rms) noise ranges from $\sim 3 \mathrm{mK}$ to $\sim 10 \mathrm{mK}$ per velocity channel in antenna temperature units $\left(T_{\mathrm{A}}^{*}\right)$. Because of the relatively compact angular size of the $\mathrm{C}^{+}-$and $\mathrm{S}^{+}$-emitting layers (compared to the secondary beam lobes), we converted the $T_{\mathrm{A}}^{*}$ scale to main beam temperature, $T_{\mathrm{MB}}=T_{\mathrm{A}}^{*} \cdot \eta_{\mathrm{F}} / \eta_{\mathrm{MB}}$, where $\eta_{\mathrm{F}}$ is the forward efficiency and $\eta_{\mathrm{MB}}$ the main beam efficiency. We

\footnotetext{
2 In $\mathrm{H}$ II regions and IFs, $\mathrm{S}^{+}$can be detected through the infrared ${ }^{2} \mathrm{P}-$ ${ }^{2} D$ lines at $\sim 1.03 \mu \mathrm{m}$ (Walmsley et al. 2000) and in the visible through the ${ }^{2} D-{ }^{4} S$ lines at $6718,6733 \AA$ (Pellegrini et al. 2009). The estimation of [S] from these lines is sensitive to $T_{\mathrm{e}}$ gradients, extinction, and ionization corrections (Rudolph et al. 2006). The ground ${ }^{4} S$ and excited ${ }^{2} D$ electronic states are separated by $\sim 21400 \mathrm{~K}$. In PDRs and cold molecular clouds, $\mathrm{S}^{+}$largely exists in the ${ }^{4} S$ state.

Carbon and sulfur RRLs were identified in the early 1970s. These detections implied the presence of what at that time was called "C II and S II regions" in star-forming clouds. The term "PDR" started to be used in the late 1980s to model these FUV-illuminated regions.
}

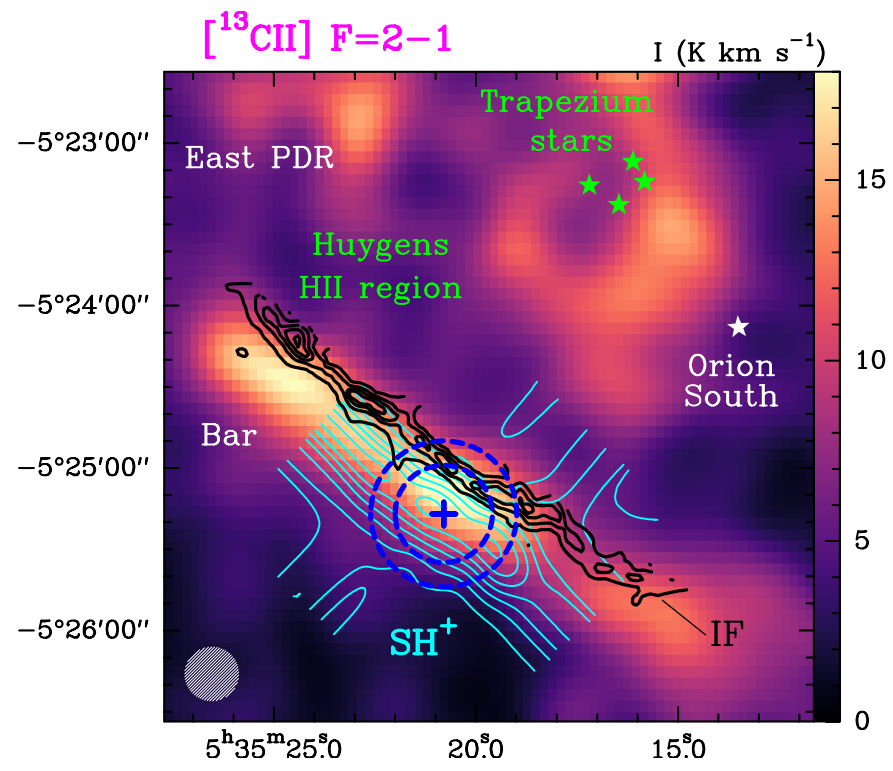

Fig. 1. Multiple FUV-illuminated edges in OMC-1 (dense PDRs). The colored map shows the integrated emission $(I)$ of the $\left[{ }^{13} \mathrm{C}\right.$ II $]$ $\left({ }^{2} P_{3 / 2}-{ }^{2} P_{1 / 2} F=2-1\right)$ line at $1900.466 \mathrm{GHz}$ (Goicoechea et al. 2015). This emission traces the $\mathrm{C}^{+}$layers of the molecular cloud. Cyan contours show a map of the $\mathrm{SH}^{+} 1_{0}-0_{1} F=1 / 2-3 / 2$ emission at $345.944 \mathrm{GHz}$ (Goicoechea et al. 2021), a proxy of the $\mathrm{S}^{+}$layer (both maps at $\left.\theta_{\mathrm{HPBW}} \simeq 20^{\prime \prime}\right)$. Black contours show the O I $1.317 \mu \mathrm{m}$ fluorescent emission that delineates the IF of the Bar (Walmsley et al. 2000). Dashed blue circles show the smallest and largest $\theta_{\mathrm{HPBW}}$ of our Yebes $40 \mathrm{~m}$ observations toward the DF position: $36^{\prime \prime}$ and 54", respectively.

adopted the values tabulated in Tercero et al. (2021): for example, $\eta_{\mathrm{F}} \simeq 0.92$ and $\eta_{\mathrm{MB}} \simeq 0.52$ at $\sim 40 \mathrm{GHz}$. We identified nine Cn $\alpha$ RRLs (Table B.1) that show a fainter feature at slightly higher frequency, which we assigned to Sn $\alpha$ RRLs. Figure B.1 shows the individual spectra.

\section{The $\mathrm{C}^{+}$and $\mathrm{S}^{+}$layers in the Bar PDR}

Figure 1 shows the environment around the targeted position in the Bar. The colored map shows the ${ }^{2} P_{3 / 2}-{ }^{2} P_{1 / 2}$ finestructure line emission ( $F=2-1$ hyperfine component) from the ${ }^{13} \mathrm{C}^{+}$isotope, hereafter the $\left[{ }^{13} \mathrm{C}\right.$ II $] 158 \mu \mathrm{m}$ line. While the $\left[{ }^{12} \mathrm{C}\right.$ II $] 158 \mu \mathrm{m}$ line is moderately optically thick in dense PDRs (e.g., Ossenkopf et al. 2013), $\left[{ }^{13} \mathrm{C}\right.$ II $]$ lines are optically thin and trace the distribution of dense PDRs at the FUV-illuminated rims of OMC-1 (Goicoechea et al. 2015). The " $\mathrm{C}^{+}$layer" (where most of the gas-phase carbon is in the form of $\mathrm{C}^{+}$) extends from the ionization front (IF; the $\mathrm{H}^{+}-\mathrm{H}$ transition at the $\mathrm{H}$ II regionPDR interface) to the PDR layers, where most of the gas-phase carbon is converted first into $\mathrm{C}$ atoms and then into $\mathrm{CO}$, at a few magnitudes of visual extinction into the cloud $\left(A_{V}\right)$. The distance between the IF and $A_{V}(\mathrm{CO})$, perpendicular to the Bar and in the UV-illumination direction, is $\sim 15^{\prime \prime}(\sim 0.03 \mathrm{pc}$; Tielens et al. 1993; Goicoechea et al. 2016). Interestingly, the distribution of the C91 $\alpha$ emission, mapped with the Very Large Array (VLA; Wyrowski et al. 1997), is remarkably similar to the $v=1-0 S(1)$ $\mathrm{H}_{2}$ emission that traces the $\mathrm{H}-\mathrm{H}_{2}$ transition at the DF.

Although $\mathrm{S}$ has a lower IP than $\mathrm{C}$, the extent of the $\mathrm{S}^{+}$layer depends on the wavelength- and $A_{V}$-dependent attenuation of the FUV field (Goicoechea \& Le Bourlot 2007) as well as on details of the $\mathrm{S}$ photoionization and $\mathrm{S}^{+}$radiative recombination (RR) 


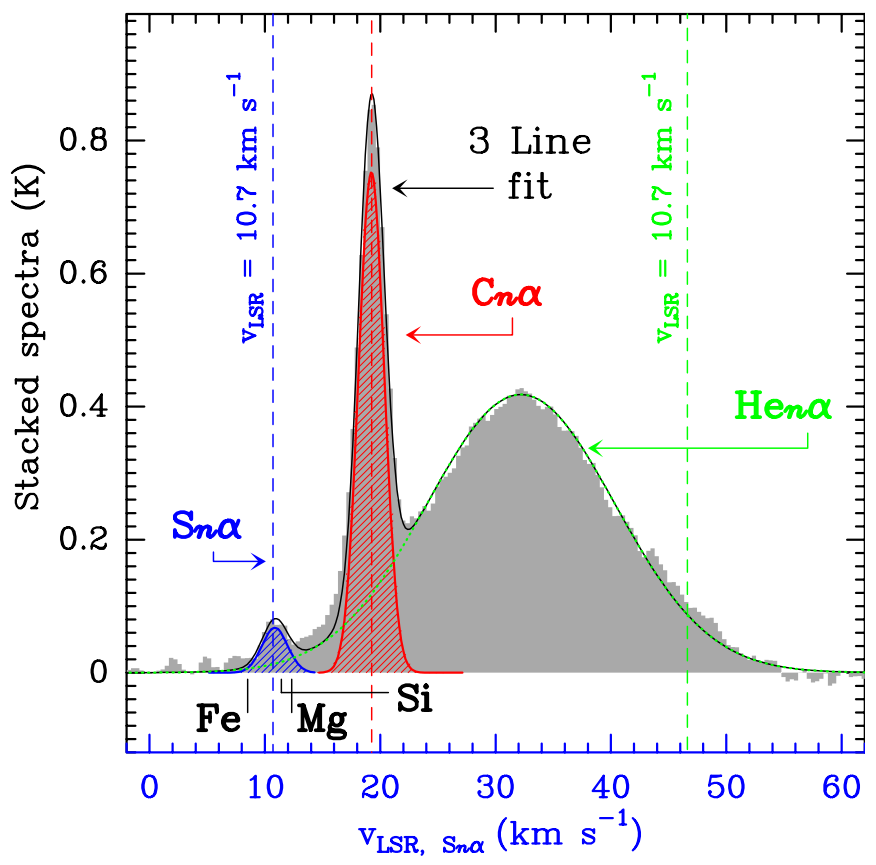

Fig. 2. Stacked spectrum (filled gray histogram) of several $n \alpha$ RRLs $(n=54-59)$. The spectrum is centered at the frequencies of Sn $\alpha$ lines and shows the expected position of $\mathrm{Mg}, \mathrm{Si}$, and Fe $n \alpha$ RRLs. The black curve is a three-Gaussian line fit: Sn $\alpha$ (blue), Cn $\alpha$ (red), and Hen $\alpha$ (green). Vertical dashed lines mark the LSR velocity of the Bar PDR.

and resonant dielectronic recombination (DR) processes. Paradoxically, a modern treatment of $\mathrm{S}^{+}$recombination, especially at low $T_{\mathrm{e}}$, is still lacking (Badnell 1991; Bryans et al. 2009). Our observations encompass the $\mathrm{C}^{+}$and $\mathrm{S}^{+}$layers of the Bar. Indeed, Fig. 1 shows that the observed DF position coincides with the position of the $\mathrm{SH}^{+}$emission peak (cyan contours). This trace molecular ion forms via gas reactions of $\mathrm{S}^{+}$with vibrationally excited $\mathrm{H}_{2}$ molecules (e.g., Goicoechea et al. 2021).

\section{Results: Detection of Sn $\alpha$ RRLs}

We detected new Cn $\alpha$ and Hen $\alpha$ RRLs (from $n=51$ to 59; Fig. B.1). Helium RRLs arise from the adjacent Huygens H II region $\left(\mathrm{IP}_{\mathrm{He}}=24.6 \mathrm{eV}\right)$ and from the different foreground layers of ionized gas in Orion's Veil (O'Dell 2001). The high electron temperature and pressure in the hot and fully ionized gas produce the broad line width $\left(\Delta v \simeq 20 \mathrm{~km} \mathrm{~s}^{-1}\right)$ of He RRLs. Consistent with ionized gas that flows toward the observer, He RRLs are blueshifted by $\sim 15 \mathrm{~km} \mathrm{~s}^{-1}$ with respect to OMC emission.

A visual inspection of each individual $\mathrm{C} n \alpha$ spectrum shows the presence of a narrow emission feature at a slightly higher frequency. Unless produced by a molecular line ${ }^{4}$, these features imply the presence of an RRL from an element heavier than ${ }^{12} \mathrm{C}(12 \mathrm{amu})$. To determine their origin, we calculated the $n \alpha$ RRL frequencies of ${ }^{32} \mathrm{~S}(31.972 \mathrm{amu})$ using the Rydberg equation and assuming that the recombined electron in level $n$ moves on the field of a nucleus of effective charge $Z_{\text {eff }} \simeq 1=16\left(p^{+}\right)-15$ (inner $e^{-}$). This means that RRLs of (manyelectron) neutral atoms possess frequencies close to hydrogen RRLs but, as the mass of the nucleus increases, shifted to slightly higher frequencies (Table B.1). Likewise, we computed the RRL

\footnotetext{
4 None of the Sn $\alpha$ line frequencies correspond to molecules present in the Bar (Cuadrado et al. 2015, 2017) or in molecular-line catalogs.
}

frequencies of other neutral atoms with low IP $(<13.6 \mathrm{eV})$ and with solar abundances $[\mathrm{X}]_{\odot}>10^{-5}:{ }^{24} \mathrm{Mg}(23.985 \mathrm{amu}),{ }^{28} \mathrm{Si}$ (27.977 amu), and ${ }^{56} \mathrm{Fe}$ (55.935 amu).

In order to increase the signal-to-noise ratio $(\mathrm{S} / \mathrm{N})$ of the detection, and because RRLs of similar $n$ and $\Delta n$ have similar emission properties, we stacked all $\mathrm{C} n \alpha$ spectra from $n=54$ to 59 (those with the highest $\mathrm{S} / \mathrm{N}$ ). We first converted each spectrum to the local standard of rest (LSR) velocity scale, then resampled them to the same velocity channel resolution, and finally stacked them. The resulting spectrum in Fig. 2 displays three line features that can be fitted with three Gaussians reasonably well: a broad line from Hen $\alpha$ RRLs and two narrow lines, one from Cn $\alpha$ RRLs and another from the heavier element. According to the atomic mass differences between carbon and $\mathrm{S}, \mathrm{Mg}, \mathrm{Si}$, and $\mathrm{Fe}$, the velocity separation of their $n \alpha$ RRLs from $\mathrm{C} n \alpha$ lines is: $\quad \delta v\left({ }^{12} \mathrm{C}-{ }^{24} \mathrm{Mg}\right)=-6.9 \mathrm{~km} \mathrm{~s}^{-1}, \quad \delta v\left({ }^{12} \mathrm{C}-{ }^{28} \mathrm{Si}\right)=-7.8 \mathrm{~km} \mathrm{~s}^{-1}$, $\delta v\left({ }^{12} \mathrm{C}-{ }^{32} \mathrm{~S}\right)=-8.6 \mathrm{~km} \mathrm{~s}^{-1}$, and $\delta v\left({ }^{12} \mathrm{C}-{ }^{56} \mathrm{Fe}\right)=-10.8 \mathrm{~km} \mathrm{~s}^{-1}$. The observed (peak-to-peak) velocity separation between the $\mathrm{Cn} \alpha$ and the higher-frequency feature in the Bar is $\delta v=-8.4 \pm 0.3 \mathrm{~km} \mathrm{~s}^{-1}$. Therefore, the third feature is largely produced by Sn $\alpha$ RRLs. This conclusion is supported by the severe depletions of $\mathrm{Mg}, \mathrm{Si}$, and $\mathrm{Fe}$ inferred in diffuse neutral clouds (e.g., Jenkins 2009). The typical depletion ${ }^{1}$ factors in these clouds are $D(\mathrm{Mg}) \simeq-1.3, D(\mathrm{Si}) \simeq-1.4$, and $D(\mathrm{Fe}) \simeq-2.2 \mathrm{dex}$ (Savage \& Sembach 1996). This implies $[\mathrm{S}]>[\mathrm{Mg}] \simeq[\mathrm{Si}]>[\mathrm{Fe}]$ (in the gas phase). It also means that in interstellar PDRs, RRLs of these more condensable elements are much fainter than those of S. From our observations we can only conclude that $\mathrm{Mg}, \mathrm{Si}$, and $\mathrm{Fe}$ are depleted by more than -0.6 dex relative to $\mathrm{S}$.

The stacked Sn $\alpha$ line shows a similar Gaussian profile, line width $\left(\Delta v \simeq 2.6 \pm 0.2 \mathrm{~km} \mathrm{~s}^{-1}\right.$; mostly microturbulent broadening), and velocity centroid $\left(v_{\mathrm{LSR}} \simeq 10.8 \pm 0.1 \mathrm{~km} \mathrm{~s}^{-1}\right)$ as those of $\mathrm{C} 54 \alpha,\left[{ }^{13} \mathrm{C} \mathrm{II}\right]$, and $\mathrm{SH}^{+}$lines - all observed toward the DF position at $\theta_{\mathrm{HPBW}} \simeq 45^{\prime \prime}$ (see Fig. C.1). The narrow line profiles (see Table C.1) demonstrate that Sn $\alpha$ RRLs arise from PDR gas and not from the fully ionized H II region.

\section{Discussion}

To guide our interpretation, Fig. 3 shows the $\left[\mathrm{C}^{+}\right],[\mathrm{C}],[\mathrm{CO}]$, $\left[\mathrm{S}^{+}\right],[\mathrm{S}],\left[\mathrm{H}_{2} \mathrm{~S}\right.$-ice $]$, and $\left[e^{-}\right]$abundance profiles predicted by a homogeneous PDR model ${ }^{5}$ run with the Meudon code (Le Petit et al. 2006). We adopted the gas-phase carbon abundance, $[\mathrm{C}]_{\text {Ori }}=(1.4 \pm 0.6) \cdot 10^{-4}$, measured from UV absorptionline observations of the translucent neutral gas toward the Trapezium star $\theta^{1}$ Ori B (Sofia et al. 2004). This abundance implies a depletion factor ${ }^{1}$ of $D(\mathrm{C}) \simeq-0.3$ dex (carbon that is incorporated into carbonaceous grains, polycyclic aromatic hydrocarbons, and macromolecules such as fullerenes; e.g., Berné \& Tielens 2012). We assumed that this is precisely the initial gas-phase carbon abundance in the Bar (i.e., $\left[\mathrm{C}^{+}\right]_{\mathrm{IF}}=[\mathrm{C}]_{\text {Ori }}$ ). Figure 3 shows that the $\mathrm{C}^{+}$abundance remains constant in the $\mathrm{C}^{+}$ layer, from the $\operatorname{IF}\left(A_{V}=0\right)$ to $A_{V}(\mathrm{C} \mathrm{I}) \simeq 3$. The $\mathrm{S}^{+}$layer (where most of the sulfur is in the form of $\mathrm{S}^{+}$) extends a bit deeper, up to $A_{V} \simeq 4$. However, one would need observations at high

\footnotetext{
5 We adopted a uniform gas density $n_{\mathrm{H}}=n(\mathrm{H})+2 n\left(\mathrm{H}_{2}\right)=5 \cdot 10^{4} \mathrm{~cm}^{-3}$, an FUV radiation field of $\chi_{\mathrm{FUV}}=10^{4}$ times the Draine's field, and $R_{V}=A_{V} / E_{B-V}=5.5$, appropriate for Orion. This is sometimes called the "interclump" medium of the Bar (e.g., Young Owl et al. 2000). This model is valid for the outer $\mathrm{C}^{+}$layer and reproduces the observed IF$A_{V}(\mathrm{CO})$ angular separation. The chemical network includes updated gas-phase and grain-surface reactions (Goicoechea et al. 2021).
} 


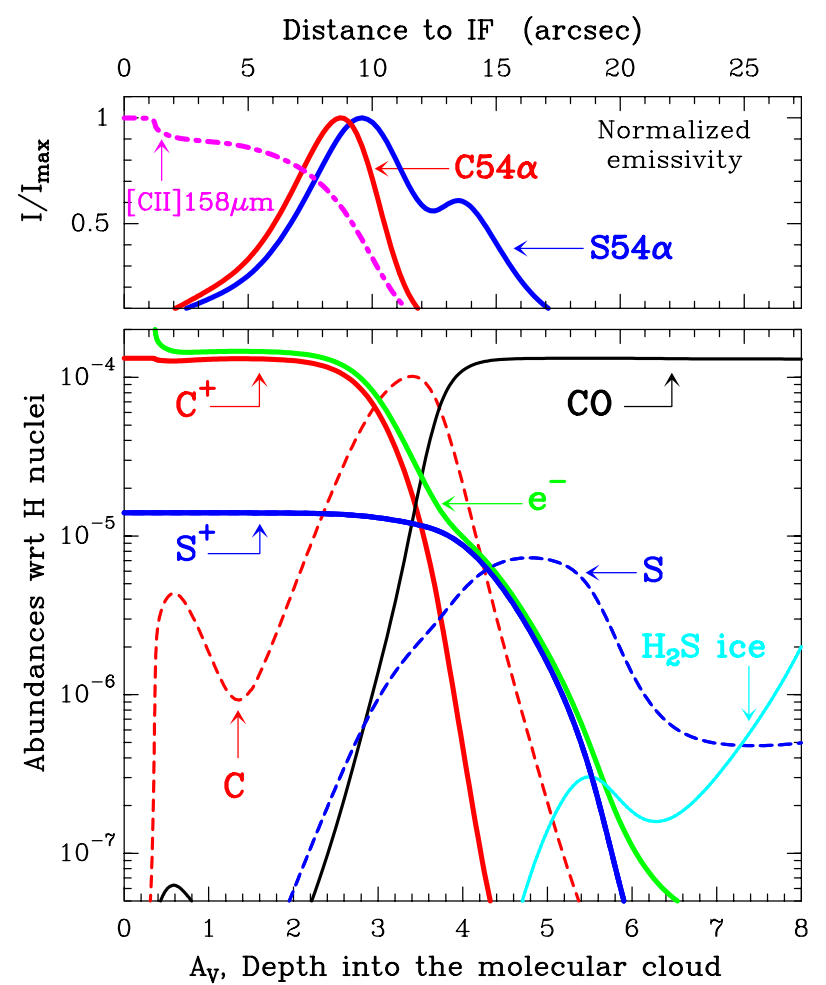

Fig. 3. Model $^{5}$ of the $\mathrm{C}^{+}$layer with $[\mathrm{C}] /[\mathrm{S}]=10$. Lower panel: abundance profiles as a function of $A_{V}$ along the UV-illumination direction: from the IF to the cloud interior (lower axis) and the equivalent angular distance perpendicular to the Bar (upper axis). Upper panel: normalized emissivities of the [C II] $158 \mu \mathrm{m}$, S54 $\alpha$, and C54 $\alpha$ lines. For the RRLs, we adopted an LTE model (see Appendix A).

angular resolution $\left(\leqslant 5^{\prime \prime}\right)$ to spatially resolve the different sizes of the $\mathrm{C}^{+}$and $\mathrm{S}^{+}$layers; they are small at the moderate gas densities of the Bar. In these intermediate PDR layers, $A_{V} \simeq 3.5$ to 5.5 , photoionization of $\mathrm{S}$ atoms becomes the major source of $e^{-}$, and thus of the ionization fraction. This is one of the reasons why determining $[\mathrm{S}]$ in dense gas is relevant.

Because the HPBW of the $40 \mathrm{~m}$ telescope encompasses the $\mathrm{C}^{+}$and $\mathrm{S}^{+}$layers of the Bar, and assuming that the $\mathrm{C}$ and $\mathrm{S}$ level populations of fairly high principal quantum numbers $n$ depart from local thermodynamic equilibrium (LTE) in a similar fashion (e.g., Dupree 1974; Salgado et al. 2017), we can determine the gas-phase carbon-to-sulfur abundance ratio, $[\mathrm{C}] /[\mathrm{S}]=n_{\mathrm{C}^{+}} / n_{\mathrm{S}^{+}}$, directly from the $\mathrm{C} n \alpha / \mathrm{S} n \alpha=R_{n \alpha}$ line intensity ratio. From the stacked spectrum we obtain $R_{n \alpha}=10.4 \pm 0.6$. This is a factor of two lower than the $[\mathrm{C}]_{\odot} /[\mathrm{S}]_{\odot}$ ratio. As we adopted $\left[\mathrm{C}^{+}\right]_{\mathrm{IF}}=[\mathrm{C}]_{\text {Ori }}$, the $R_{n \alpha}$ ratio leads to $\left[\mathrm{S}^{+}\right]_{\mathrm{IF}}=[\mathrm{S}]_{\text {Ori }}=(1.4 \pm 0.4) \cdot 10^{-5}$

Under the above assumptions, the initial [S] in the OMC matches the solar abundance. Interestingly, it also coincides with the stellar [S] abundances measured in young B-type stars of the Ori OB1 association (Daflon et al. 2009). This result implies very little depletion of $S$ in dense clouds, $D(S)=0.0 \pm 0.2$. In the unlikely case that the $\left[\mathrm{C}^{+}\right]_{\mathrm{IF}}$ in the Bar is significantly lower than the value measured by Sofia et al. (2004), our estimated $[\mathrm{S}]_{\text {Ori }}$ abundance would be an upper limit and $D(\mathrm{~S})$ would decrease accordingly. Still, an LTE excitation model with $N\left(\mathrm{~S}^{+}\right) \simeq 7 \cdot 10^{17} \mathrm{~cm}^{-2}$ (Appendix A) is consistent with the absolute intensities of the observed sulfur RRLs, with $[\mathrm{S}]_{\text {Ori }}=1.4 \cdot 10^{-5}$, and with $N\left(\mathrm{C}^{+}\right) / N\left(\mathrm{~S}^{+}\right) \simeq 10$.
The ratio $R_{(54-59) \alpha}$ observed in the Bar is higher than that determined at several-arcminute resolution in the pioneering cm-wave observations of the $\rho$ Oph dark cloud $\left(R_{158 \alpha} \simeq 3.5\right.$ and $R_{110 \alpha} \simeq 6.2$; Pankonin \& Walmsley 1978) and the W3A and Orion B clouds $\left(R_{158 \alpha} \simeq 3.1\right.$ and 7.1, respectively; Chaisson et al. 1972). These variations can be produced by (i) the different beam filling factors of the $\mathrm{C}^{+}$- and $\mathrm{S}^{+}$-emitting regions in these clouds, such that their RRL emission is not spatially resolved and the large beam mixes various PDRs, (ii) very different excitation properties of $\mathrm{S} n \alpha$ versus Cn $\alpha$ RRLs, or (iii) intrinsically lower $[\mathrm{C}] /[\mathrm{S}]$ ratios. We currently favor possibility (i). Indeed, our higher angular resolution observations nearly spatially resolve the Bar PDR. If this were not the case, and if the $\mathrm{S}^{+}$layer were significantly more extended than the $\mathrm{C}^{+}$ layer, then the intrinsic $R_{(54-59) \alpha}$ ratios would be larger. In the future, more sensitive observations and more details on the RR and DR of $\mathrm{S}^{+}$ions will allow us to model the non-LTE excitation of sulfur RRLs individually, as well as to constrain $n_{\mathrm{e}}$ and $T_{\mathrm{e}}$ as a function of $A_{V}$ (e.g., RRLs do not arise from the same [C II] $158 \mu \mathrm{m}$-emitting layer; see Fig. 3). In the meantime, ours is probably the most direct estimation of $[\mathrm{S}]$ in a dense cloud.

Since stellar sulfur abundances increase with increasing metallicity (e.g., Perdigon et al. 2021), and the estimated [S] in stars and $\mathrm{H}$ II regions decreases with galactocentric radius $\left(R_{\mathrm{GC}}\right)$ as -0.04 dex kpc $^{-1}$ (e.g., Rudolph et al. 2006; Daflon et al. 2009; Arellano-Córdova et al. 2020), we anticipate shallow variations of [S] in molecular clouds of different $R_{\mathrm{GC}}$. A related open question (e.g., Sofia 2004) is how to reconcile the low sulfur depletion in the ISM with the existence of interplanetary dust particles containing sulfur (Bradley 1994) and with the existence of solid $\mathrm{MgS}$ in evolved stars. The latter seems only viable if $\mathrm{MgS}$ is present in the outer coating surfaces of circumstellar grains and not in their cores (Lombaert et al. 2012; Sloan et al. 2014). These outer surfaces may be more easily destroyed in the harsh ISM. Observations of Sn $\alpha$ RRLs from a larger sample of starforming regions will improve our understanding of interstellar sulfur.

Acknowledgements. We thank J. H. Black for reminding us that our spectra of the Orion Bar may contain sulfur RRLs and for useful remarks on how $\mathrm{S}^{+}$ dielectronic capture may proceed. We thank B. Tercero and the Yebes staff for their help with these observations. We thank the Spanish MCIYU for funding support under grants PID2019-106110GB-I00 and AYA2016-75066-C2-1-P.

\section{References}

Arellano-Córdova, K. Z., Esteban, C., García-Rojas, J., \& Méndez-Delgado, J. E. 2020, MNRAS, 496, 1051

Asplund, M., Grevesse, N., Sauval, A. J., \& Scott, P. 2009, ARA\&A, 47, 481 Badnell, N. R. 1991, ApJ, 379, 356

Ball, J. A., Cesarsky, D., Dupree, A. K., Goldberg, L., \& Lilley, A. E. 1970, ApJ, 162, L25

Berné, O., \& Tielens, A. G. G. M. 2012, Proc. Nat. Acad. Sci., 109, 401

Bradley, J. P. 1994, Science, 265, 925

Brocklehurst, M., \& Seaton, M. J. 1971, Astrophys. Lett., 9, 139

Bryans, P., Kreckel, H., Roueff, E., Wakelam, V., \& Savin, D. W. 2009, ApJ, 694, 286

Chaisson, E. J., \& Lada, C. J. 1974, ApJ, 189, 227

Chaisson, E. J., Black, J. H., Dupree, A. K., \& Cesarsky, D. A. 1972, ApJ, 173, L131

Churchwell, E., Smith, L. F., Mathis, J., Mezger, P. G., \& Huchtmeier, W. 1978, A\&A, 70, 719

Cuadrado, S., Goicoechea, J. R., Pilleri, P., et al. 2015, A\&A, 575, A82

Cuadrado, S., Goicoechea, J. R., Cernicharo, J., et al. 2017, A\&A, 603, A124

Cuadrado, S., Salas, P., Goicoechea, J. R., et al. 2019, A\&A, 625, L3

Daflon, S., Cunha, K., de la Reza, R., Holtzman, J., \& Chiappini, C. 2009, AJ, 138,1577

Draine, B. T. 2003, ARA\&A, 41, 241 
J. R. Goicoechea and S. Cuadrado: The sulfur abundance in the Orion Molecular Cloud

Dupree, A. K. 1974, ApJ, 187, 25

Falgarone, E., Cesarsky, D. A., Encrenaz, P. J., \& Lucas, R. 1978, A\&A, 65, L13

Federman, S. R., Sheffer, Y., Lambert, D. L., \& Gilliland, R. L. 1993, ApJ, 413, L51

Fitzpatrick, E. L., \& Spitzer, L. J. 1994, ApJ, 427, 232

Fuente, A., Goicoechea, J. R., Pety, J., et al. 2017, ApJ, 851, L49

Genzel, R., \& Stutzki, J. 1989, ARA\&A, 27, 41

Goicoechea, J. R., \& Le Bourlot, J. 2007, A\&A, 467, 1

Goicoechea, J. R., Teyssier, D., Etxaluze, M., et al. 2015, ApJ, 812, 75

Goicoechea, J. R., Pety, J., Cuadrado, S., et al. 2016, Nature, 537, 207

Goicoechea, J. R., Aguado, A., Cuadrado, S., et al. 2021, A\&A, 647, A10

Hollenbach, D. J., \& Tielens, A. G. G. M. 1999, Rev. Mod. Phys., 71, 173

Howk, J. C., Sembach, K. R., \& Savage, B. D. 2006, ApJ, 637, 333

Jenkins, E. B. 2009, ApJ, 700, 1299

Le Petit, F., Nehmé, C., Le Bourlot, J., \& Roueff, E. 2006, ApJS, 164, 506

Lombaert, R., de Vries, B. L., de Koter, A., et al. 2012, A\&A, 544, L18

Mathis, J. S. 1990, ARA\&A, 28, 37

Natta, A., Walmsley, C. M., \& Tielens, A. G. G. M. 1994, ApJ, 428, 209

O'Dell, C. R. 2001, ARA\&A, 39, 99

Ossenkopf, V., Röllig, M., Neufeld, D. A., et al. 2013, A\&A, 550, A57

Pabst, C. H. M., Goicoechea, J. R., Teyssier, D., et al. 2020, A\&A, 639, A2

Pankonin, V., \& Walmsley, C. M. 1978, A\&A, 64, 333

Pellegrini, E. W., Baldwin, J. A., Ferland, G. J., Shaw, G., \& Heathcote, S. 2009 ApJ, 693, 285
Perdigon, J., de Laverny, P., Recio-Blanco, A., et al. 2021, A\&A, in press, https://doi .org/10.1051/0004-6361/202040147

Rudolph, A. L., Fich, M., Bell, G. R., et al. 2006, ApJS, 162, 346

Salas, P., Oonk, J. B. R., Emig, K. L., et al. 2019, A\&A, 626, A70

Salgado, F., Morabito, L. K., Oonk, J. B. R., et al. 2017, ApJ, 837, 141

Savage, B. D., \& Sembach, K. R. 1996, ARA\&A, 34, 279

Shingledecker, C. N., Lamberts, T., Laas, J. C., et al. 2020, ApJ, 888, 52

Silverglate, P. R. 1984, ApJ, 278, 604

Sloan, G. C., Lagadec, E., Zijlstra, A. A., et al. 2014, ApJ, 791, 28

Smirnov, G. T., Sorochenko, R. L., \& Walmsley, C. M. 1995, A\&A, 300, 923

Sofia, U. J. 2004, in Astrophysics of Dust, eds. A. N. Witt, G. C. Clayton, \& B. T. Draine, ASP Conf. Ser., 309, 393

Sofia, U. J., Lauroesch, J. T., Meyer, D. M., \& Cartledge, S. I. B. 2004, ApJ, 605, 272

Tercero, F., López-Pérez, J. A., Gallego, J. D., et al. 2021, A\&A, 645, A37

Tielens, A. G. G. M., Meixner, M. M., van der Werf, P. P., et al. 1993, Science, 262, 86

Vallee, J. P. 1989, ApJ, 341, 238

Walmsley, C. M., Natta, A., Oliva, E., \& Testi, L. 2000, A\&A, 364, 301

Wyrowski, F., Schilke, P., Hofner, P., \& Walmsley, C. M. 1997, ApJ, 487, L171

Young Owl, R. C., Meixner, M. M., Wolfire, M., Tielens, A. G. G. M., \& Tauber, J. 2000, ApJ, 540, 886 


\section{Appendix A: Absolute intensities of sulfur RRLs}

The level populations of the recombined $\mathrm{S}$ atom can be written as $N(n)=b_{n} N(n)_{\text {LTE }}$, where $n$ is the principal quantum number, $N(n)_{\text {LTE }}$ are the LTE populations given by the Saha-Boltzmann equation, and $b_{n}$ are the so-called departure coefficients. We consider that the S54 $\alpha$ RRL emission is dominated by spontaneous emission, with little contribution from stimulated emission. Hence, we neglected amplification of any background continuum emission $\left(T_{\mathrm{c}}\right)$. This is a reasonable assumption for these RRLs in Orion (e.g., Natta et al. 1994). Indeed, the $7 \mathrm{~mm}$ continuum emission is produced by the cosmic microwave background, the long-wave tail of the dust thermal emission, and the free-free Bremsstrahlung emission. In Orion, the last component largely arises from the Huygens H II region, but this nebular emission is in the foreground relative to the Bar. In addition, owing to the low electron densities in PDRs, we estimate that the free-free opacity is very low, $\tau_{\mathrm{ff}}(7 \mathrm{~mm}) \sim 10^{-7}$. Hence, the continuum opacity $\left(\tau_{\mathrm{c}}=\tau_{\mathrm{ff}}+\tau_{\mathrm{d}}\right)$ is dominated by the opacity of warm $\left(T_{\mathrm{d}} \simeq 50 \mathrm{~K}\right)$ dust grains, which is also low, $\tau_{\mathrm{d}}(7 \mathrm{~mm}) \sim 10^{-5}$. The observed sulfur RRLs are optically thin $\left(\tau_{\mathrm{RRL}}\right.$ of a few $\left.10^{-4}\right)$ and are in the Rayleigh-Jeans regime (with $h v / k \ll T_{\mathrm{e}}$ and $\left.T_{\mathrm{c}} \ll T_{\mathrm{e}}\right)$. Hence, we can write the S54 $\alpha$ integrated line intensity $\left(I_{\mathrm{S} 54 \alpha}\right.$ in $\left.\mathrm{K} \mathrm{km} \mathrm{s}^{-1}\right)$ as:

$I_{\mathrm{S} 54 \alpha}=\int T_{\mathrm{MB}} \mathrm{d} v \simeq 15 b_{54+1} T_{\mathrm{e}}^{-1.5} \exp \left(\chi_{54}\right) E M_{\mathrm{S}^{+}}$,

where $\chi_{n}=15.79 \cdot 10^{4} n^{-2} T_{\mathrm{e}}^{-1}$ and $E M_{\mathrm{S}^{+}}=n_{\mathrm{e}} n_{\mathrm{S}^{+}} L\left(\right.$ in $\left.\mathrm{pc} \mathrm{cm}^{-6}\right)$ is the $\mathrm{S}^{+}$emission measure along a slab of thickness $L$ (along the line-of-sight; $L$ should not be confused with the cloud depth in the UV-illumination direction). The factor $\simeq 15$ is specific to $54 \alpha$ RRLs and includes the oscillator strength $\Delta n M(\Delta n) \simeq 0.1908$ for $\Delta n=1$ (Brocklehurst \& Seaton 1971; Dupree 1974; Salas et al. 2019). When excitation conditions are close to LTE and as the principal quantum number $n$ increases, the departure coefficients approach $b_{n} \simeq 1$. Coefficients for hydrogen and carbon RRLs have been estimated in the literature. At the relatively low electron temperatures of a PDR, these coefficients are typically $0.3<b_{n}<1$, with values up to $b_{n} \simeq 1.5$ when the details of resonant dielectronic capture for non-hydrogenic atoms are included (e.g., Salgado et al. 2017). In this study we implicitly assumed that any substantial difference between $\mathrm{S}^{+}$and $\mathrm{C}^{+}$recombination will have little effect on the relative intensities of their moderately high- $n$ recombination spectra. Nonetheless, we point out again that the low $T_{\mathrm{e}} \mathrm{RR}$ and DR of $\mathrm{S}^{+}$have not been properly studied (Bryans et al. 2009). Here, we simply adopted $b_{n}=1$ and $T_{\mathrm{e}}=T_{\text {gas }}$ (i.e., LTE) to estimate the RRL emissivities (see the PDR model in Fig. 3).

We then calculated the expected intensity of the S54 $\alpha$ RRL using Eq. (A.1) and compared it with the observational value in the Orion Bar PDR, $I_{\mathrm{S} 54 \alpha} \simeq I_{\mathrm{C} 54 \alpha} / R_{(54-59) \alpha}$, where $R_{(54-59) \alpha}$ is the $\mathrm{C}(54-59) \alpha / \mathrm{S}(54-59) \alpha$ integrated line intensity ratio of the stacked spectrum (see Table C.1). We obtain $I_{\mathrm{S} 54 \alpha}($ observations $)=0.407 / 10.4=0.04 \mathrm{~K} \mathrm{~km} \mathrm{~s}^{-1}$. Figure A. 1 shows a grid of single-slab models appropriate for the two possible sources of RRL emission discussed in the literature of the Bar: the "interclump" medium model - with $n_{\mathrm{e}} \simeq 10 \mathrm{~cm}^{-3}$ and $L \simeq 0.2 \mathrm{pc}$ - and the denser "clump" or "high-pressure" model - with $n_{\mathrm{e}} \simeq 100 \mathrm{~cm}^{-3}$ and $L \simeq 0.025 \mathrm{pc}$. Assuming a beam filling factor of one, both models reproduce the observed $I_{\mathrm{S} 54 \alpha}$ intensities with a similar column $N\left(\mathrm{~S}^{+}\right) \simeq 7 \cdot 10^{17} \mathrm{~cm}^{-2}$ but different (LTE) electron temperatures: $T_{\mathrm{e}}$ (interclump) $\simeq 125 \mathrm{~K}$ and $T_{\mathrm{e}}($ clump $) \simeq 500 \mathrm{~K}$. Cuadrado et al. (2019) recently reported the detection of $3 \mathrm{~mm}$ wave carbon RRLs in the Orion Bar:
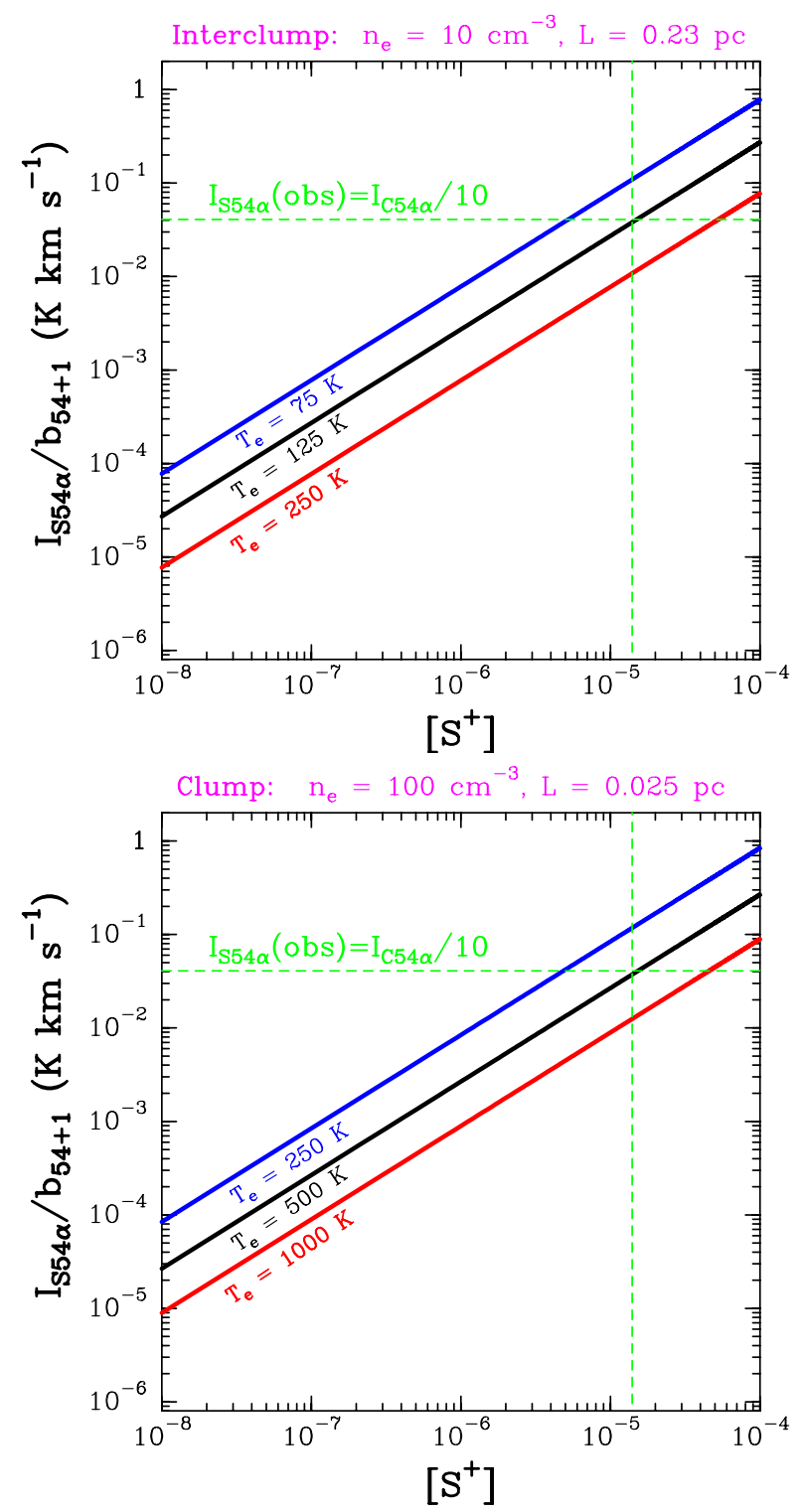

Fig. A.1. S54 $\alpha$ intensity as a function of sulfur abundance and $T_{\mathrm{e}}$. Upper panel: interclump model of the Bar. Lower panel: model that assumes that RRLs arise from denser clumps or high-pressure gas (see text). The horizontal dashed green line marks the observational value.

$\alpha$ lines from $n=42$ to $38, \beta(\Delta n=2)$ lines, and $\gamma(\Delta n=3)$ lines. Although they estimated, from single-slab models, that these lines arise from PDR layers with $n_{\mathrm{e}} \simeq 60-100 \mathrm{~cm}^{-3}$ and $T_{\mathrm{e}} \simeq 500-600 \mathrm{~K}$, the clump versus interclump dichotomy is not yet fully solved (Tielens et al. 1993; Goicoechea et al. 2016). Since PDRs have steep temperature and density gradients, we suspect that only higher angular resolution observations will clarify the exact small spatial-scale origin of these lines.

The modeled column density of $\mathrm{S}^{+}$, beam-averaged over the mean angular resolution of the Yebes $40 \mathrm{~m}$ observations $\left(\sim 45^{\prime \prime}\right)$, is a factor of ten lower than the $N\left(\mathrm{C}^{+}\right)$independently inferred from maps of the $\left[{ }^{13} \mathrm{C}\right.$ II $] 158 \mu \mathrm{m}$ line emission toward the same angular area of the Bar: $N\left(\mathrm{C}^{+}\right) \simeq 7 \cdot 10^{18} \mathrm{~cm}^{-2}$ (see Table 2 of Ossenkopf et al. 2013). Therefore, the observed S(5459) $\alpha$ line intensities are consistent with both $N\left(\mathrm{C}^{+}\right) / N\left(\mathrm{~S}^{+}\right) \simeq 10$ and with $\left[\mathrm{S}^{+}\right] \simeq 1.4 \cdot 10^{-5}$. More refined estimations of $T_{e}$ and $n_{e}$ will require a proper non-LTE model, higher angular resolution observations, and higher $\mathrm{S} / \mathrm{N}$ detections of several RRLs. 


\section{Appendix B: Carbon RRL observational parameters and sulfur RRL frequencies}
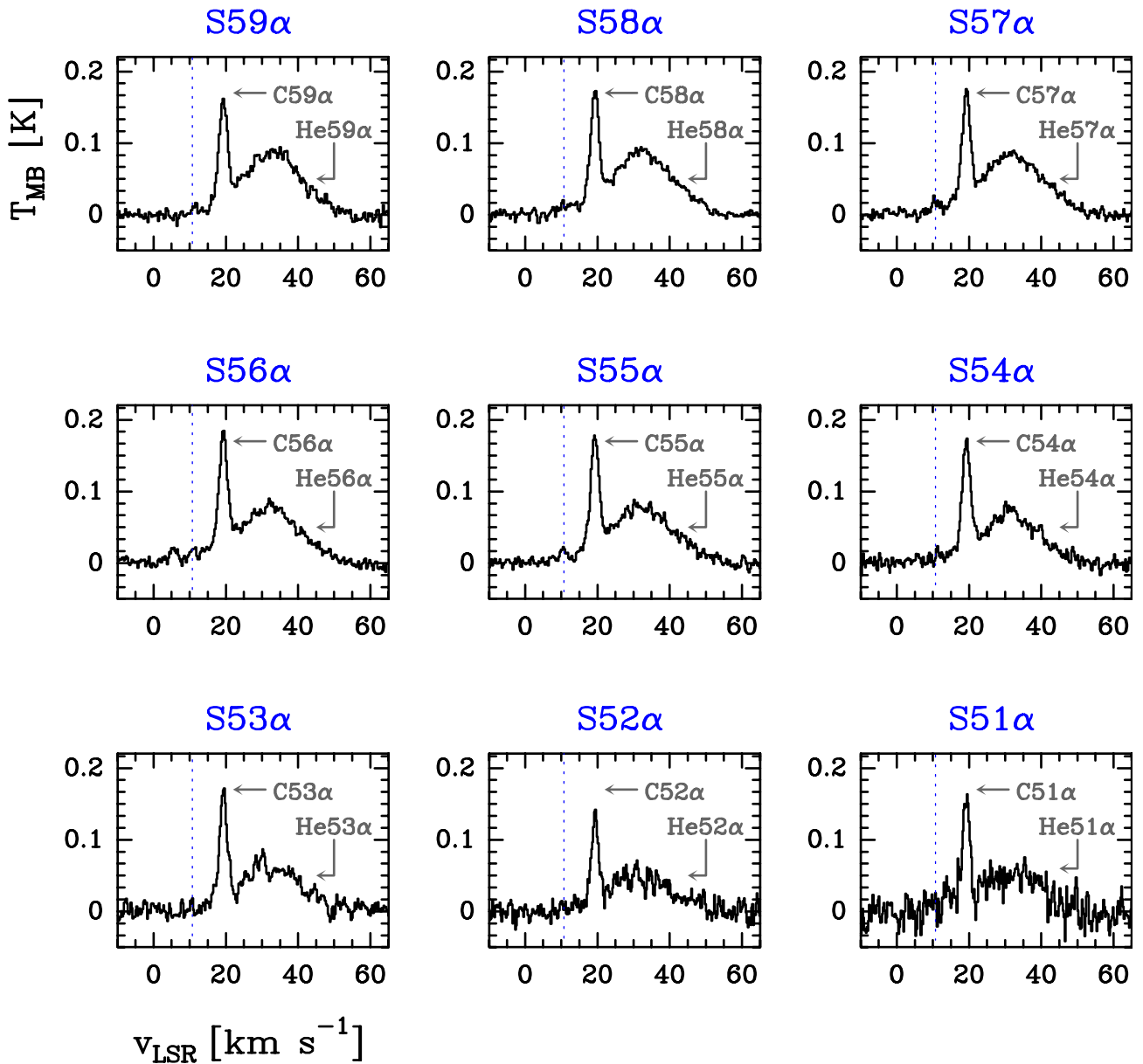

Fig. B.1. Sulfur, carbon, and helium RRLs observed with the Yebes $40 \mathrm{~m}$ radio telescope between $31 \mathrm{GHz}$ and $48 \mathrm{GHz}$ (at $38 \mathrm{kHz}$ resolution) toward the Orion Bar. Dashed lines indicate the position of Sn $\alpha$ RRLs at $v_{\mathrm{LSR}} \simeq 10.7 \mathrm{~km} \mathrm{~s}^{-1}$, the emission of the neutral PDR gas.

Table B.1. Cn $\alpha$ line spectroscopic parameters obtained from a two-Gaussian line fit to C and He RRLs.

\begin{tabular}{lcccccccc}
\hline \hline Line & $\begin{array}{c}\text { Frequency } \\
{[\mathrm{GHz}]}\end{array}$ & $\begin{array}{c}I=\int_{\left.\mathrm{K} \mathrm{km} \mathrm{s}^{-1}\right]} T_{\mathrm{MB}{ }^{(a)}} \\
\end{array}$ & $\begin{array}{c}v_{\mathrm{LSR}} \\
{\left[\mathrm{km} \mathrm{s}^{-1}\right]}\end{array}$ & $\begin{array}{c}\Delta v \\
{\left[\mathrm{~km} \mathrm{~s}^{-1}\right]}\end{array}$ & $\begin{array}{c}T_{\mathrm{MB}, \text { Peak }}{ }^{(a)} \\
{[\mathrm{K}]}\end{array}$ & $\begin{array}{c}S / N^{(b)} \\
\text { Line }\end{array}$ & $\begin{array}{c}\text { Frequency } \\
{[\mathrm{GHz}]}\end{array}$ \\
\hline $\mathrm{C} 59 \alpha$ & 31.23889 & $0.410(0.011)$ & $10.7(0.1)$ & $2.8(0.1)$ & 0.139 & 25 & $\mathrm{~S} 59 \alpha$ & 31.23978 \\
$\mathrm{C} 58 \alpha$ & 32.86859 & $0.404(0.009)$ & $10.7(0.1)$ & $2.6(0.1)$ & 0.146 & 32 & $\mathrm{~S} 58 \alpha$ & 32.86953 \\
$\mathrm{C} 57 \alpha$ & 34.61364 & $0.378(0.009)$ & $10.7(0.1)$ & $2.5(0.1)$ & 0.141 & 29 & $\mathrm{~S} 57 \alpha$ & 34.61463 \\
$\mathrm{C} 56 \alpha$ & 36.48445 & $0.399(0.011)$ & $10.7(0.1)$ & $2.6(0.1)$ & 0.147 & 30 & $\mathrm{~S} 56 \alpha$ & 36.48550 \\
$\mathrm{C} 55 \alpha$ & 38.49255 & $0.402(0.010)$ & $10.7(0.1)$ & $2.6(0.1)$ & 0.146 & 26 & $\mathrm{~S} 55 \alpha$ & 38.49365 \\
$\mathrm{C} 54 \alpha$ & 40.65077 & $0.407(0.013)$ & $10.7(0.1)$ & $2.6(0.1)$ & 0.146 & 22 & $\mathrm{~S} 54 \alpha$ & 40.65193 \\
$\mathrm{C} 53 \alpha$ & 42.97340 & $0.377(0.022)$ & $10.7(0.1)$ & $2.5(0.1)$ & 0.145 & 18 & $\mathrm{~S} 53 \alpha$ & 42.97463 \\
$\mathrm{C} 52 \alpha$ & 45.47640 & $0.260(0.012)$ & $10.7(0.1)$ & $2.2(0.1)$ & 0.112 & 12 & $\mathrm{~S} 52 \alpha$ & 45.47770 \\
$\mathrm{C} 51 \alpha$ & 48.17762 & $0.319(0.023)$ & $10.6(0.1)$ & $2.2(0.2)$ & 0.138 & 8 & $\mathrm{~S} 51 \alpha$ & 48.17900 \\
\hline
\end{tabular}

Notes. The last column provides the frequencies of the Sn $\alpha$ RRLs. ${ }^{(a)}$ Intensities in main beam temperature. ${ }^{(b)}$ S/N determined from the line emission peak at a spectral resolution of $38 \mathrm{kHz}$. Parentheses indicate the uncertainty obtained by the Gaussian fitting routine. 


\section{Appendix C: Line parameters of the stacked} spectrum and comparison with other lines

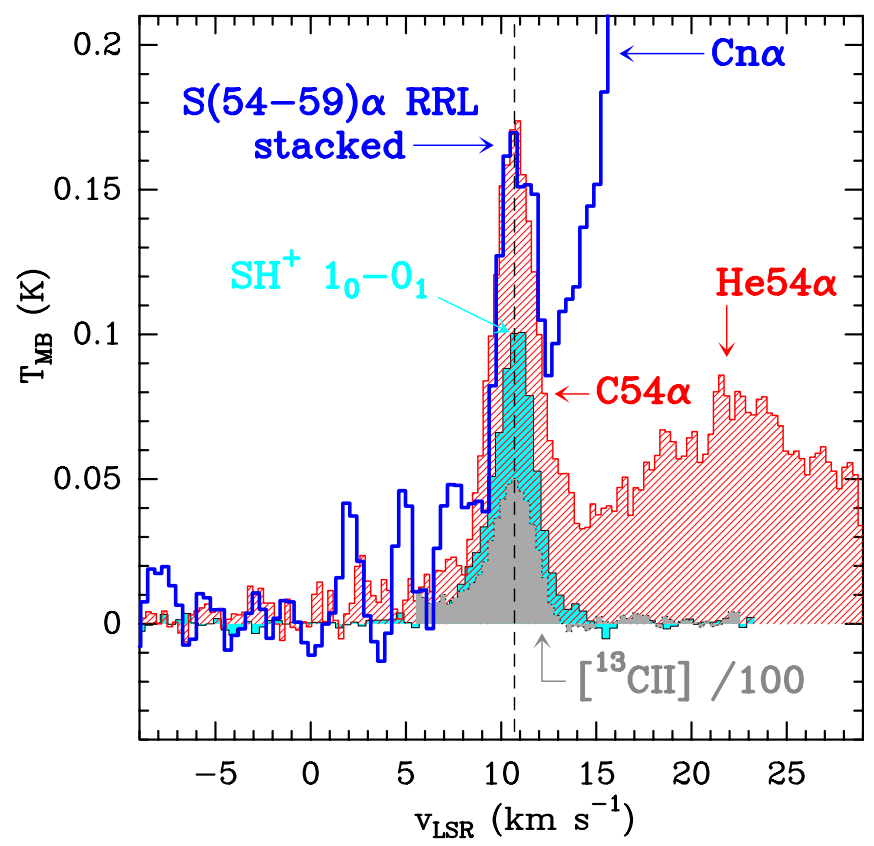

Fig. C.1. Similar line profiles, velocity centroids, and line widths of the stacked Sn $\alpha$ line (blue), C54 $\alpha$ (hatched red), $\left[{ }^{13} \mathrm{C}\right.$ II $] 158 \mu \mathrm{m}$ $\left(F=2-1\right.$; solid gray), and $\mathrm{SH}^{+}$(hatched cyan) lines toward the DF position (all observed or extracted at $\theta_{\mathrm{HPBW}} \simeq 45^{\prime \prime}$ and $\simeq 0.3 \mathrm{~km} \mathrm{~s}^{-1}$ resolutions). The dashed line corresponds to the LSR velocity of the PDR, $v_{\mathrm{LSR}}=10.7 \mathrm{~km} \mathrm{~s}^{-1}$.

Table C.1. Line spectroscopic parameters obtained from Gaussian fits toward the Orion Bar PDR.

\begin{tabular}{lccccccc}
\hline \hline Species & Line & $\begin{array}{c}\text { Frequency } \\
{[\mathrm{GHz}]}\end{array}$ & $\begin{array}{c}\theta_{\mathrm{HPBW}} \\
{\left[{ }^{\prime \prime}\right]}\end{array}$ & $\begin{array}{c}I=\int_{\mathrm{MB}} \mathrm{d} v \\
{\left[\mathrm{~K} \mathrm{~km} \mathrm{~s}^{-1}\right]}\end{array}$ & $\begin{array}{c}v_{\mathrm{LSR}} \\
{\left[\mathrm{km} \mathrm{s}^{-1}\right]}\end{array}$ & $\begin{array}{c}\Delta v \\
{\left[\mathrm{~km} \mathrm{~s}^{-1}\right]}\end{array}$ & $\begin{array}{c}T_{\mathrm{MB}, \text { Peak }} \\
{[\mathrm{K}]}\end{array}$ \\
\hline $\mathrm{C}$ & $(54-59) \alpha$ & Stacked & & $2.084(0.012)$ & $10.6(0.1)$ & $2.6(0.1)$ & 0.753 \\
$\mathrm{~S}$ & $(54-59) \alpha$ & Stacked & & $0.200(0.011)$ & $10.8(0.1)$ & $2.6(0.2)$ & 0.072 \\
\hline $\mathrm{SH}^{+}$ & $1_{0}-0_{1} F=1 / 2-3 / 2$ & 345.94438 & $45^{(a)}$ & $0.274(0.003)$ & $10.6(0.1)$ & $2.6(0.1)$ & 0.097 \\
${ }^{13} \mathrm{C}^{+}$ & ${ }^{2} P_{3 / 2}-{ }^{3} P_{1 / 2} F=2-1$ & 1900.46610 & $45^{(a)}$ & $12.888(0.812)$ & $10.6(0.1)$ & $2.6(0.2)$ & 4.707 \\
\hline $\mathrm{C}$ & $54 \alpha$ & 40.65077 & 45 & $0.407(0.013)$ & $10.7(0.1)$ & $2.6(0.1)$ & 0.146 \\
$\mathrm{He}$ & $54 \alpha$ & 40.64706 & 45 & $1.408(0.021)$ & $-4.7(0.3)$ & $19.5(0.5)$ & 0.069 \\
\hline
\end{tabular}

Notes. ${ }^{(a)}$ Extracted from $\left[{ }^{13} \mathrm{CII}\right]$ (Goicoechea et al. 2015) and $\mathrm{SH}^{+}$(Goicoechea et al. 2021) maps smoothed to $\theta_{\mathrm{HPBW}}=45^{\prime \prime}$ resolution (shown in Fig. 1). Parentheses indicate the uncertainty obtained by the Gaussian fitting routine. 